\title{
Relevance of clinical trial results in myocardial infarction to medical practice: comparison of four year outcome in participants of a thrombolytic trial, patients receiving routine thrombolysis, and those deemed ineligible for thrombolysis
}

\author{
N Brown, M Melville, D Gray, T Young, A M Skene, R G Wilcox, J R Hampton
}

\begin{abstract}
Objective-To assess the medium to long term outcome of patients ineligible for thrombolysis compared to those enrolled in a clinical trial of thrombolysis and patients receiving non-trial thrombolysis. Design-Cohort study based on the Nottingham heart attack register.

Setting-Two district general hospitals serving a defined urban/rural population. Subjects-All patients admitted with a confirmed acute myocardial infarction during 1992 categorised as either participants of a thrombolytic trial (group $A, n=$ 140), receiving non-trial thrombolysis (group B, $\mathbf{n}=329$ ), or deemed ineligible for lytic treatment (group $C, n=431$ ).

Main outcome measures-Background characteristics, inhospital treatment, patterns of follow up, referrals to cardiologists, revascularisation rates, and short and long term survival.
\end{abstract}

Results-Clinical trial recruits were younger by almost 10 years, were less likely to have a previous history of myocardial infarction, and more likely to be in Killip class 1 on admission than those ineligible for thrombolysis. Cardiology follow up was mandatory for all surviving trial participants but $22 \%$ of patients in group $B$ and $31 \%$ of patients in group C received no follow up, and during four years less than $50 \%$ ever saw a cardiologist. Revascularisation was performed in $17.2 \%$ of patients in group $A, 13.6 \%$ of patients in group $B$, and $7.5 \%$ of patients in group C. Cumulative mortality at a median of four years was $24.3 \%$ in group $A, 36.8 \%$ in $B$, and $59.6 \%$ in group $C$. Adjusting for age, sex, previous myocardial infarction, type of infarction, and Killip class in a logistic regression model the odds ratios (OR) of death at four years for groups $B$ and $C$ were 1.60 (95\% confidence intervals $(\mathrm{CI}) 0.97$ to $2.63, \mathrm{p}=0.065$ ) and $2.64(95 \%$ CI 1.61 to $4.32, p<0.001)$, respectively, when compared to group A (OR 1).

Conclusions-Patients enrolled into thrombolytic trials are at low risk. Patients deemed ineligible for thrombolysis are high risk, receive less surveillance, are less likely to be revascularised or receive trial proven treatments, have a poor long term outcome not entirely explained by increased age or severity of infarction, and deserve further evaluation.

\section{(Heart 1999;81:598-602)}

Keywords: myocardial infarction; thrombolysis; clinical trials

An overview of the randomised clinical trials of thrombolysis in patients with acute myocardial infarction has reported mortality rates at 35 days of $9.6 \%$ in treated patients versus $11.5 \%$ in controls. ${ }^{1}$ All trials have specific inclusion and exclusion criteria and selection may favour recruitment of those with a low mortality risk. Consequently, those recruited into trials may not be representative of all patients managed by participating hospitals.

Patients from the Nottingham hospitals were included in two trials of thrombolysis during $1992^{23}$ and the inhospital mortality of patients in each of these was very similar to the published reports. However, the introduction of thrombolysis into routine clinical practice has not led to a decline in mortality in Nottingham, which has remained persistently high. ${ }^{4}$

Which patients are eligible for thrombolytic treatment depends upon entry criteria if a clinical trial is in progress, or on local protocols for non-trial patients. The proportion of patients who might be eligible for thrombolysis has been estimated at $50 \% .^{5}$ Patients who do not receive thrombolysis are a heterogeneous group who may be considered unsuitable for thrombolysis because they present outside the conventional "time window" for treatment, have a recognised contraindication, have a normal 12 lead ECG on arrival in hospital, or because their infarction is not recognised.

Few studies have reviewed the outcome in those deemed ineligible for thrombolytic treatment, but those that have showed a notably worse short term outcome with inhospital or 35 day mortality rates between $16.8 \%$ and $27 \%$. $^{6-8}$

The aim of this study was to determine the medium to long term outcome of an unselected population of patients presenting to Nottingham's two hospitals, comparing those deemed ineligible for thrombolysis with those enrolled into a clinical trial of thrombolysis or given thrombolysis in accordance with the coronary care unit protocols. We assessed patterns of
Dr Brown. 29 January 1999 
Table 1 Comparison of baseline characteristics of patients with acute myocardial infarction in 1992 in Nottingham enrolled into a clinical trial (group A), given standard thrombolysis (group B), or deemed ineligible for lytic treatment (group C)

\begin{tabular}{|c|c|c|c|c|c|c|c|}
\hline & \multicolumn{2}{|c|}{$\begin{array}{l}\text { Group A } \\
(n=140)\end{array}$} & \multicolumn{2}{|c|}{$\begin{array}{l}\text { Group B } \\
(n=329)\end{array}$} & \multicolumn{2}{|c|}{$\begin{array}{l}\text { Group C } \\
(n=431)\end{array}$} & \multirow{2}{*}{$\begin{array}{l}p \text { value } \\
<0.001\end{array}$} \\
\hline Mean (SD) age (years) & 61.5 & $(11.0)$ & 64. & $(11.0)$ & 70. & $(11.3)$ & \\
\hline Male & 96 & $(68.6)$ & 220 & $(66.9)$ & 245 & $(56.8)$ & 0.005 \\
\hline Previous myocardial infarction & 18 & (12.9) & 54 & $(16.4)$ & 109 & (25.3) & 0.001 \\
\hline Previous coronary revascularisation & 3 & $(2.1)$ & 2 & $(0.6)$ & 7 & $(1.6)$ & - \\
\hline Killip class 1 & 85 & $(60.7)$ & 202 & $(61.4)$ & 220 & $(51.0)$ & 0.009 \\
\hline Killip class 2 & 45 & $(32.1)$ & 109 & $(33.1)$ & 138 & $(32.0)$ & 0.946 \\
\hline Killip class $3 / 4$ & 9 & $(6.4)$ & 18 & $(5.5)$ & 70 & $(16.2)$ & $<0.001$ \\
\hline $\mathrm{Q}$ wave infarction & 125 & $(89.3)$ & 265 & $(80.5)$ & 255 & $(59.2)$ & $<0.01$ \\
\hline Anterior infarct & 63 & $(45.0)$ & 131 & $(39.8)$ & 192 & $(44.5)$ & 0.367 \\
\hline Management on a coronary care unit & 140 & $(100)$ & 329 & $(100)$ & 246 & $(57.0)$ & $<0.001$ \\
\hline Initial management by cardiologist & 38 & $(27.1)$ & 70 & $(21.3)$ & 120 & $(27.8)$ & 0.103 \\
\hline Inhospital mortality & 12 & $(8.6)$ & 43 & (13.1) & 150 & $(34.8)$ & $<0.001$ \\
\hline
\end{tabular}

Values are numbers (\%).

follow up, the use of health service resources, coronary interventions, and long term survival.

\section{Methods}

The methods of data collection for the Nottingham heart attack register have been described in detail elsewhere. ${ }^{9}$ The entire cohort of patients admitted to the two Nottingham hospitals with a documented myocardial infarct in 1992 were identified on admission and detailed hospital data recorded. Post-discharge data were obtained from hospital and general practitioner records to the end of July 1996. All patients were included irrespective of whether they were admitted to the coronary care unit or to a general medical ward.

The diagnosis of acute myocardial infarction was based on a compatible history plus either serial changes in the ECG of ST elevation evolving new $Q$ waves or a rise in cardiac enzymes to more than twice the upper limit of normal, or both.

We assessed patterns of follow up, outpatient attendances, readmissions, reinfarctions, drug treatment, morbidity, and the use of cardiac procedures (including exercise electrocardiography, echocardiography, and coronary angiography). Referral to a cardiologist was documented along with rates of coronary angioplasty and cardiac surgery.

All four year survivors in the cohort were traced and mailed a questionnaire containing basic demographic questions, and a modified Rose angina and dyspnoea questionnaire. ${ }^{10}$

Patients were categorised as: having taken part in either of two thrombolytic trials conducted at some time in 1992 (the global utilisation of streptokinase and tissue plasminogen activator for occluded coronary arteries trial (GUSTO) ${ }^{3}$ or the late assessment of thrombolytic efficacy study (LATE) ${ }^{2}$ ) (group A); having received thrombolysis in accordance with the coronary care unit protocols (group $\mathrm{B})$; and having received all necessary care excluding thrombolysis (group C).

Mortality and cause of death was established through review of hospital notes, postmortem records, coroner's office records, and the Office of National Statistics.

STATISTICAL ANALYSIS

Differences in selected characteristics were analysed using the $\chi^{2}$ test. Actuarial survival curves were generated using Cox regression. ${ }^{11}$ Crude odds ratios (OR) for long term mortality were calculated using group $\mathrm{A}$ as the control group (OR 1) and then adjusted for age, sex, previous myocardial infarction, type of infarction, and Killip class on admission in a logistic regression model. All tests of significance were two tailed with $\mathrm{p}<0.05$ considered significant. The S-PLUS ${ }^{12}$ statistical package was used for all analysis.

\section{Results}

During 1992 there were 960 admissions with proven myocardial infarction, 21 of whom were reinfarctions and for survival purposes counted from the first event only. Thirty nine patients were excluded from this analysis as they were non-district, temporary residents or miscodes. Of the survivors to discharge $(n=695)$ follow up data were obtained on all but one patient.

\section{CLINICAL CHARACTERISTICS}

The characteristics of patients enrolled into a clinical trial or given thrombolysis in accordance with our local protocol differed significantly from non-thrombolysed patients (table 1). Patients recruited into trials (group A) were significantly younger by almost 10 years than those deemed ineligible for thrombolysis (group C). A lower proportion of patients entered into a thrombolytic trial (group A) or receiving non-trial thrombolysis (group B) had a history of previous myocardial infarction $(\mathrm{p}<0.001$ and $\mathrm{p}=0.004$, respectively) and fewer were in Killip class 3 and 4 ( $p=0.003$ and $p=0.005$, respectively) than those deemed ineligible for thrombolysis (group C). Patients in group A were slightly younger than those in group $B(p=0.003)$ but there were no other significant differences in characteristics between these patients.

There was no significant difference in the proportion of patients initially managed by a cardiologist.

REASONS FOR INELIGIBILITY FOR THROMBOLYSIS IN GROUP C

Patients were deemed ineligible for thrombolysis $(n=431)$ on the basis of contraindications to treatment in $45(10.4 \%)$, lack of ECG changes in $22(5.1 \%)$, late arrival in 150 $(34.8 \%)$, and multiple reasons in 141 (32.7\%). No reason for ineligibility was apparent in 73 patients $(16.9 \%)$.

\section{MANAGEMENT AND INHOSPITAL MORTALITY}

The lowest inhospital mortality was observed in patients recruited into the clinical trials, as shown in table 1. Mortality in those given "routine" thrombolysis was slightly higher although this was not significant $(p=0.219)$. Mortality in those patients who did not receive thrombolysis (group C) was significantly higher than that for patients in either groups $\mathrm{A}$ or B $(\mathrm{p}<0.001)$.

Table 2 shows inhospital treatment in each group. Significantly more patients recruited into a thrombolytic trial were given $\beta$ blockers compared to those ineligible for thrombolytic treatment $(p<0.001)$. The majority of pa- 
Table 2 Inhospital treatment of patients with acute myocardial infarction in 1992 in Nottingham enrolled into a clinical trial (group A), given standard thrombolysis (group $B)$, or deemed ineligible for lytic treatment (group $C$ )

\begin{tabular}{|c|c|c|c|c|}
\hline & $\begin{array}{l}\text { Group A } \\
(n=140)\end{array}$ & $\begin{array}{l}\text { Group B } \\
(n=329)\end{array}$ & $\begin{array}{l}\text { Group } C \\
(n=431)\end{array}$ & $p$ value \\
\hline Antiarrhythmics & $11(7.9)$ & $23(7.0)$ & $63(14.6)$ & 0.002 \\
\hline$\beta$ blockers & $79(56.4)$ & $160(48.6)$ & $136(31.6)$ & $<0.001$ \\
\hline Aspirin & $134(95.7)$ & $320(97.3)$ & $315(73.1)$ & $<0.001$ \\
\hline ACE inhibitors & $13(9.3)$ & $20(6.1)$ & $72(16.7)$ & $<0.001$ \\
\hline $\begin{array}{l}\text { Anticoagulants (heparin, } \\
\text { warfarin) }\end{array}$ & $122(87.1)$ & $223(67.8)$ & $171(39.7)$ & $<0.001$ \\
\hline Diuretics & $47(33.6)$ & $134(40.7)$ & $250(58.0)$ & $<0.001$ \\
\hline Digoxin & $12(8.6)$ & $31(9.4)$ & $92(21.3)$ & $<0.001$ \\
\hline
\end{tabular}

Values are numbers of patients receiving treatment (\%).

Table 3 Readmissions and use of medical resources after discharge in Nottingham in patients enrolled into a clinical trial (group A), given standard thrombolysis (group B), or deemed ineligible for lytic treatment (group $C$ )

\begin{tabular}{lcccr}
\hline & $\begin{array}{l}\text { Group } A \\
(n=128)\end{array}$ & $\begin{array}{l}\text { Group } B \\
(n=286)\end{array}$ & $\begin{array}{l}\text { Group } C \\
(n=281)\end{array}$ & p value \\
\hline $\begin{array}{l}\text { Recurrent non-fatal myocardial } \\
\quad \text { infarction }\end{array}$ & $18(14.1)$ & $32(11.2)$ & $25(8.9)$ & 0.284 \\
$\begin{array}{l}\text { Admission with angina or possible } \\
\quad \text { myocardial infarction }\end{array}$ & $28(21.9)$ & $40(14.0)$ & $41(14.6)$ & 0.101 \\
$\begin{array}{l}\text { Admission with congestive heart failure } \\
\text { Exercise test or thallium scan }\end{array}$ & $8(6.3)$ & $19(6.6)$ & $45(16.0)$ & $<0.001$ \\
Echocardiography & $28(21.9)$ & $81(28.3)$ & $33(11.7)$ & $<0.001$ \\
Coronary angiography & $44(34.4)$ & $91(31.8)$ & $112(39.9)$ & 0.129 \\
24 hour tape & $28(21.9)$ & $45(15.7)$ & $31(11.0)$ & 0.015 \\
Coronary angioplasty & $18(14.1)$ & $18(6.3)$ & $28(10.0)$ & 0.035 \\
Coronary artery bypass surgery & $4(3.1)$ & $4(1.4)$ & $2(0.7)$ & - \\
Lipid tests & $18(14.1)$ & $35(12.2)$ & $19(6.8)$ & 0.032 \\
\hline
\end{tabular}

Values are numbers of patients (\%). Median follow up four years.

tients in groups A and B were given aspirin but significantly fewer of those in group $\mathrm{C}$ received this treatment. Angiotensin converting enzyme (ACE) inhibitors, diuretics, and digoxin were more likely to be prescribed for patients in group C.

FOLLOW UP AFTER DISCHARGE AND REFERRALS TO CARDIOLOGISTS

Follow up by a cardiologist was mandatory for patients recruited into GUSTO and LATE, and 123 of $128(96.1 \%)$ of patients attended clinic in accordance with trial protocol.

Of patients cared for by cardiologists outwith a trial a follow up appointment was arranged for 125 of 141 (89\%) survivors. The remaining patients were under the care of a general physician: 223 of 286 survivors ( $78 \%$ ) who received

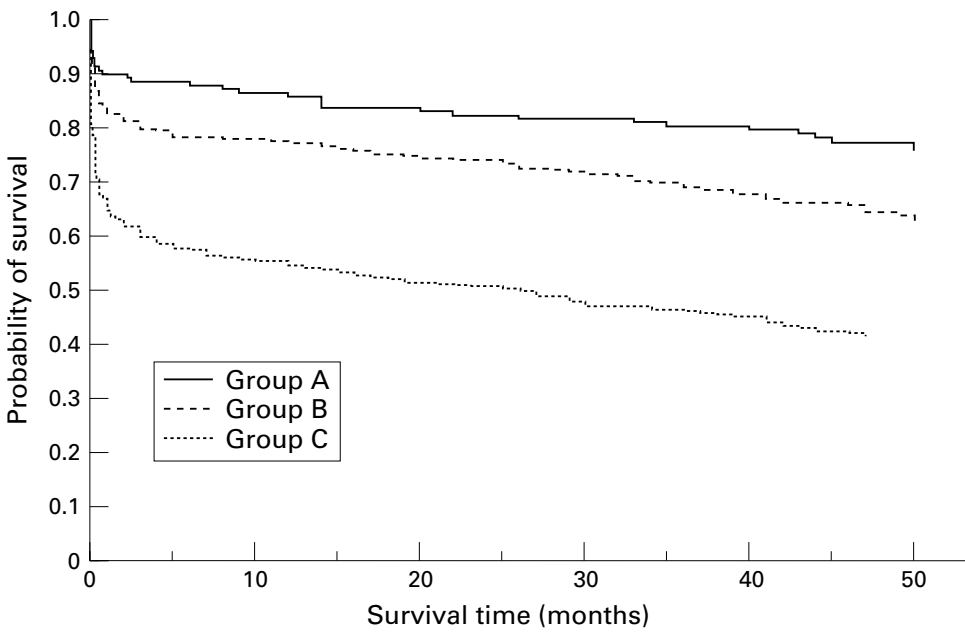

Figure 1 Unadjusted survival curves using Cox regression in patients enrolled into a thrombolytic trial (group A), those given non-trial thrombolysis (group B), and those deemed ineligible for thrombolysis (group $C$ ). non-trial thrombolysis and 193 of 281 (69\%) who did not receive a thrombolytic were given an outpatient appointment.

A further $67(23.4 \%)$ and 42 (14.9\%) patients in groups $\mathrm{B}$ and $\mathrm{C}$ were referred after discharge to a cardiologist either by the patient's general practitioner or hospital general physician.

\section{SYMPTOMS AT 4-6 WEEKS IN THOSE RECEIVING} FOLLOW UP

In patients who attended a follow up clinic there was no significant difference in the frequency of recorded angina at 4-6 weeks: 24 $(19.4 \%)$ patients in group A, $59(26.5 \%)$ in group B, and $42(21.5 \%)$ in group C. In addition there were no significant differences in the prevalence of symptomatic congestive heart failure, varying from $12.9 \%$ to $17.4 \%$.

\section{READMISSIONS AND USE OF RESOURCES AFTER} DISCHARGE

Recurrent non-fatal myocardial infarction (table 3) occurred during follow up in 18 $(14.1 \%)$ patients in group A, $32(11.2 \%)$ in group B, and $25(8.9 \%)$ in group C. There were no significant differences in admissions with angina or possible infarction in any group. More patients in group $\mathrm{C}$ were readmitted with congestive heart failure compared to patients in the other groups receiving thrombolysis.

Table 3 also shows further investigations performed following myocardial infarction. There was a greater use of exercise testing in both groups $\mathrm{A}$ and $\mathrm{B}$ compared to group C $(\mathrm{p}=0.012$ and $\mathrm{p}<0.001$, respectively) and $\mathrm{a}$ significantly greater use of coronary angiography in clinical trial patients (group A) compared to those ineligible for thrombolysis (group C). Seventeen per cent of trial recruits subsequently had coronary revascularisation, compared to $14 \%$ and $7 \%$ of patients in groups B and C, respectively. More 24 hour tapes were requested in patients in group A (14\%), and echocardiography (at least one scan) was requested in a similar proportion for each group. Lipid profiles were requested more often for patients in groups A and B than for those not receiving thrombolysis (group C).

SURVIVAL

Figure 1 shows that unadjusted survival was best in those participating in a thrombolytic trial (group A), intermediate in those given non-trial thrombolysis (group B), and worst in those deemed ineligible for thrombolysis (group C). Crude OR for death at a median of four years for groups B and C compared to A (OR 1) were 1.87 (95\% confidence intervals (CI) 1.19 to $2.93, \mathrm{p}=0.007)$ and $4.82(95 \%$ CI 3.12 to $7.44, \mathrm{p}<0.001$ ), respectively. The survival of patients in group B compared to group C was better (OR 2.58, 95\% CI 1.92 to $3.46, \mathrm{p}<0.001)$.

After adjusting for age, sex, previous myocardial infarction, type of infarction ( $Q$ versus non-Q), and Killip class, the long term survival of patients in group A was marginally better than group $\mathrm{B}$, although this did not reach significance (OR 1.60, 95\% CI 0.97 to 2.63, 
Table 4 Medical treatment at four years in survivors of acute myocardial infarction in 1992 in Nottingham enrolled into a clinical trial (group A), given standard thrombolysis (group B), or deemed ineligible for lytic treatment (group $C$ )

\begin{tabular}{lccrl}
\hline & $\begin{array}{c}\text { Group } A \\
(n=106)\end{array}$ & $\begin{array}{l}\text { Group } B \\
(n=208)\end{array}$ & $\begin{array}{c}\text { Group } C \\
(n=174)\end{array}$ & p value \\
\hline Antiarrhythmics & $3(2.8)$ & $3(1.4)$ & $6(3.4)$ & - \\
$\beta$ blockers & $41(38.7)$ & $66(31.7)$ & $45(25.9)$ & 0.078 \\
Aspirin & $92(86.8)$ & $173(83.2)$ & $131(75.3)$ & 0.036 \\
ACE inhibitors & $30(28.3)$ & $39(18.8)$ & $58(33.3)$ & 0.004 \\
Oral nitrates & $18(17.0)$ & $51(24.5)$ & $54(31.0)$ & 0.030 \\
Calcium antagonists & $24(22.6)$ & $52(25.0)$ & $44(25.3)$ & 0.869 \\
Diuretics & $30(28.3)$ & $73(35.1)$ & $83(47.7)$ & 0.003 \\
Digoxin & $5(4.7)$ & $12(5.8)$ & $15(8.6)$ & 0.367 \\
Lipid lowering agents & $9(8.5)$ & $15(7.2)$ & $9(5.2)$ & 0.531 \\
\hline
\end{tabular}

Values are numbers of patients receiving treatment (\%).

$\mathrm{p}=0.065)$. However, even after adjustment for these factors, survival in both groups $\mathrm{A}$ and $\mathrm{B}$ was notably better than in group C (OR for death $2.64,95 \%$ CI 1.61 to 4.32 , and 1.65 , $95 \%$ CI 1.18 to 2.31 , respectively). The proportion of cardiac deaths was similar: $77.3 \%$ in group A, $77.0 \%$ in group B, and $81.7 \%$ in group C.

\section{CURRENT CARDIOVASCULAR SYMPTOMS IN}

PATIENTS SURVIVING TO 1996

Both angina and dyspnoea were documented by Rose questionnaires. The overall response rate was $84.8 \%$ for the Rose angina questionnaire and $83.4 \%$ for the dyspnoea questionnaire. There were no significant demographic differences between questionnaire responders and non-responders. Twenty patients $(23.3 \%)$ in group A, $36(19.9 \%)$ in group B, and 32 $(21.8 \%)$ in group C complained of typical angina $(p=0.806)$. Thirty seven patients $(42.5 \%)$ in group A, $72(39.6 \%)$ in group B and $50(36.2 \%)$ in group $C$ had no dypsnoea according to the Rose questionnaire. Eighteen $(20.7 \%)$ and $32(36.8 \%)$ patients in group $\mathrm{A}$, $34(18.7 \%)$ and $76(41.8 \%)$ in group B, and 22 $(15.9 \%)$ and $66(47.8 \%)$ in group C had a dyspnoea grade of 1 and 2 or more, respectively, although none of these differences were significant $(\mathrm{p}=0.248$ to 0.631$)$.

\section{CURRENT MEDICATION IN PATIENTS SURVIVING}

TO 1996

Table 4 shows current drug treatment. Fewer patients in group $\mathrm{C}$ were receiving $\beta$ blockers but more were receiving diuretics. However the proportion of patients receiving ACE inhibitors in groups A and C were similar $(28.3 \%$ and $33.3 \%$, respectively), but lower in group B $(18.8 \%)$. Only eight patients in group A $(7.5 \%)$ were taking a diuretic without an $\mathrm{ACE}$ inhibitor compared with $20.7 \%$ and $23.6 \%$ of those patients in groups B and C, respectively. Lipid lowering agents were infrequently prescribed for all patients.

\section{Discussion}

The acute management and secondary prevention of myocardial infarction has changed considerably in the last 20 years as clinical trials have clarified the role of $\beta$ blockers, ${ }^{13}$ aspirin, ${ }^{1415}$ thrombolysis, ${ }^{61416}$ and ACE inhibitors. ${ }^{17}{ }^{18}$ The practice of "evidence based medicine" implies the routine use of treatments which randomised clinical trials have shown to be beneficial. This does not imply that all patients must be so treated; indeed, the results of trials may only apply to patient groups whose characteristics match the inclusion and exclusion criteria of the trials. Patients who are recruited into clinical trials are generally closely monitored both in hospital and after discharge and there is an abundance of data on their outcome. Less is known of those deemed ineligible for thrombolysis, although outcome in hospital ${ }^{6-8}$ and at one year ${ }^{19}$ is worse than in trial participants. The long term survival of these patients (when markers of infarct severity are accounted for), patterns of care, and intervention rates has not hitherto been described.

The inhospital mortality of our trial participants (group A) at $8.6 \%$ is little different from that reported in the GUSTO and LATE trials as a whole. ${ }^{23}$ However, we have now shown that the characteristics of patients admitted to the Nottingham hospitals who take part in clinical trials of thrombolysis or who are eligible for non-trial thrombolysis are quite different from those who are ineligible for thrombolytic treatment-myocardial infarction, as assessed on clinical criteria, is less extensive, patients are younger, and, perhaps most important, almost all trials require patients to be admitted within six hours of onset of chest pain.

Although the Nottingham hospitals have guidelines for the use of thrombolysis outside the context of clinical trials, there are clear differences in inhospital management among the treatment groups. During hospitalisation, thrombolysed patients were more likely to receive a $\beta$ blocker and aspirin; anticoagulants (heparin) were mandatory in the thrombolytic trials, and in 1992 heparin was prescribed as adjunctive treatment for those receiving alteplase outside trial protocols; patients ineligible for thrombolysis were more likely to be prescribed an antiarrhythmic drug, ACE inhibitor, diuretic, and digoxin. These findings are compatible with thrombolysis ineligible patients sustaining larger infarctions than other patients.

Primary percutaneous transluminal coronary angioplasty may be a valid treatment option for patients presenting "in time" with contraindications to thrombolysis, ${ }^{20}$ although only those eligible for either lysis or angioplasty have to date been the focus of randomised trials. Nevertheless, only $10 \%$ of UK hospitals have facilities for cardiac catheterisation and even fewer can provide a 24 hour service. ${ }^{21}$

In accordance with the trial protocol almost all trial patients were seen in a cardiology clinic after discharge. Follow up in non-trial patients was variable but in the four years of our study, less than half of all patients who did not take part in the trials were seen by a cardiologist at any time. Despite all of our patients having unequivocal evidence of myocardial infarction, we were surprised to find that of those managed by a general physician about a quarter of patients thrombolysed outside the clinical trial and a third of patients who did not receive thrombolysis were discharged without a single follow up visit. Protocol driven clinic 
attendance may explain why trial patients were more likely than other patients to have a variety of cardiological investigations and coronary revascularisation.

This study was not designed to show outcome differences between patients managed by cardiologists versus general physicians, ${ }^{22}{ }^{23}$ but it is interesting to note the greater surveillance, prescription of trial proven treatments, and revascularisation of those patients in group A. Based on current medical treatment at the time of our survey we have shown a less frequent use of aspirin in thrombolytic ineligible patients which is difficult to explain; even accounting for those with contraindications or intolerance, perhaps another $10 \%$ of patients could benefit from this simple treatment and seem to have been overlooked. Similarly the prescription of ACE inhibitors was as frequent in trial patients on follow up as in those deemed ineligible for thrombolysis, despite a greater prevalence of cardiac failure in the latter.

Four year mortality was slightly worse in patients receiving thrombolysis outwith a trial, although after allowing for age, sex, and markers of infarct severity this difference was not significant. Thrombolysis ineligible patients had the worst outcome and this could not be explained simply as a result of older age or more severe infarction at baseline. It is not possible to ascertain whether this difference is caused by the absence of initial lysis or subsequent less intensive treatment.

It could be argued that patients in trials are low risk and that those at greatest risk - that is, deemed ineligible for thrombolysis - are those with the most to gain and where our attention should now focus. It has been shown in the US that there may be overuse of angiography in those at low risk and underuse in patients at higher risk-age and availability of the procedure being the main determinants of cardiac catheter use after myocardial infarction. ${ }^{24}$

Our findings raise doubts about the validity of the "evidence base" for thrombolytic treatment for the generality of patients with myocardial infarction. Overall, patients ineligible for thrombolysis account for approximately $50 \%$ of a typical population with myocardial infarction, are at high risk in both the short and long term, and receive less attention than those who do receive thrombolysis. If their excess mortality is to be reduced, the future challenge for cardiologists is to develop strategies to determine how best to manage this high risk group of patients. It would be appropriate and reasonable in our current evidence based environment to conduct a series of clinical trials to determine whether different approaches to treatment, such as mandatory follow up by a cardiologist rather than a physician or early intervention with primary angioplasty, might produce a more favourable outcome. Until such trials are conducted, a more pragmatic and less costly approach would be simply to offer patients who are denied thrombolysis the same standards of care and easy access to clinical follow up, investigations, and treatment as those lower risk patients fortunate enough to receive the continued monitoring that follows recruitment into a clinical trial.

We are grateful to the general practitioners of Nottingham for their cooperation with this study. The Nottingham heart attack register has been supported by the Department of Health since its inception in 1973 .

1 Appleby P, Baigent C, Collins R, et al. Indications for fibrinolytic therapy in suspected acute myocardial infarction: collaborative overview of early mortality and major morbidity results from all randomised trials of more than 1000 patients. Lancet 1994;343:311-22.

2 The LATE Study Group. Late assessment of thrombolytic efficacy (LATE) study with alteplase 6-24 hours after onset of acute myocardial infarction. Lancet 1993;342:759-66.

3 The GUSTO Investigators. An international randomized trial comparing four thrombolytic strategies for acute myocardial infarction. N Engl f Med 1993;329:673-82.

4 Brown N, Young T, Gray D, et al. Inpatient deaths from acute myocardial infarction, 1982-92: analysis of data in the Nottingham heart attack register. BMF 1997;315:15964.

5 French JK, Williams BF, Hart HH, et al. Prospective evaluation of eligibility for thrombolytic therapy in acute ation of eligibility for thrombolytic therapy

6 Wilcox RG, Olsson CG, Skene AM, et al. Trial of tissue plasminogen activator for mortality reduction in acute myocardial infarction: Anglo-Scandinavian study of early hrombolysis (ASSET). Lancet 1988;2:525-30

7 Ozbek C, Heisel A, Krause M, et al. Comparison of mortality from acute myocardial infarction in patients receiving anistreplase with those not receiving thrombolysis. Am $\mathcal{F}$ Cardiol 1995; 76:1103-7.

8 Jha P, Deboer D, Sykora K, et al. Characteristics and mortality outcomes of thrombolysis trial participants and nonparticipants: a population-based comparison. $\mathrm{f} \mathrm{Am} \mathrm{Coll}$ Cardiol 1996;27:1335-42.

9 Gray D, Keating NA, Murdock J, et al. Impact of hospital thrombolysis policy on out-of-hospital response to suspected myocardial infarction. Lancet 1993;341:654-7.

10 Rose GA. Cardiovascular survey methods, 2nd ed. Geneva: World Health Organization, 1982.

11 Cox DR, Oaks D. Analysis of survival data. London: Chapman and Hall, 1984 .

12 S-plus guide to statistical and mathematical analysis, version 3.2. Seattle: Statistical Sciences, 1993.

13 Yusuf S, Peto R, Lewis J, et al. Beta blockade during and after myocardial infarction: an overview of the randomized trials. Prog Cardiovasc Dis 1985;27:335-71.

14 Second International Study of Infarct Survival Collaborative Group. Randomised trial of intravenous streptokinase, oral aspirin, both, or neither among 17,187 cases of suspected acute myocardial infarction: ISIS-2. Lancet 1988;ii:349-60

15 Antiplatelet Trialists' Collaboration. Secondary prevention of vascular disease by prolonged antiplatelet treatment. BMF 1988;296:320-31.

16 GISSI. Effectiveness of intravenous thrombolytic treatment in acute myocardial infarction. Lancet 1986;i:397-402.

17 The Acute Infarction Ramipril Efficacy (AIRE) Study Investigators. Effect of ramipril on mortality and morbidity Investigators. Effect of ramipril on mortality and morbidity
of survivors of acute myocardial infarction with clinical eviof survivors of acute myocardial infarction with
dence of heart failure. Lancet 1993;342:821-8.

18 Pfeffer MA, Braunwald E, Moye LA, et al. Effect of captopril on mortality and morbidity in patients with left ventricular dysfunction after myocardial infarction: results of the survival and ventricular enlargement trial. The SAVE investigators. N Engl F Med 1992;327:669-77.

19 Behar S, Gottlieb S, Hod H, et al. The outcome of patients with acute myocardial infarction ineligible for thrombolytic therapy. Israeli thrombolytic survey group. $\mathrm{Am} \mathcal{F} \mathrm{Med}$ 1996;101:184-91.

20 Himbert D, Juliard JM, Steg PG, et al. Primary coronary angioplasty for acute myocardial infarction with contraindication to thrombolysis. Am f Cardiol 1993;71:377-81.

21 Boyle RM. Immediate angioplasty in the United Kingdom. Br Heart f 1995;73:413-14.

22 Jollis JG, DeLong ER, Peterson ED, et al. Outcome of acute myocardial infarction according to the specialty of the admitting physician. $N$ Engl f Med 1996;335:1880-7.

23 Nash IS, Nash DB, Fuster V. Do cardiologists do it better? $\mathcal{F}$ Am Coll Cardiol 1997;29:475-8.

24 Pilote L, Miller DP, Califf RM, et al. Determinants of the use of coronary angiography and revascularization after thrombolysis for acute myocardial infarction. $N$ Engl f Med 1996;335:1198-205. 